\title{
Surgical management of retroperitoneal sarcoma and opportunities for global collaboration
}

\author{
Jennifer Wang ${ }^{1}$, Valerie P. Grignol ${ }^{2}$, Alessandro Gronchi ${ }^{3}$, Cheng-Hua Luo ${ }^{4}$, Raphael E. Pollock ${ }^{2}$, William \\ W. Tseng ${ }^{1}$
}

${ }^{1}$ Department of Surgery, Section of Surgical Oncology, University of Southern California, Keck School of Medicine, Los Angeles, CA, USA; ${ }^{2}$ Division of Surgical Oncology, Ohio State University, The James Comprehensive Cancer Center, Columbus, OH, USA; ${ }^{3}$ Department of Surgery, Fondazione IRCCS, Istituto Nazionale dei Tumori, Milan, Italy; ${ }^{4}$ Division of Surgery, Department of Retroperitoneal Tumor Surgery, Peking University International Hospital, Beijing 100871, China

Contributions: (I) Conception and design: WW Tseng; (II) Administrative support: None; (III) Provision of study materials or patients: None; (IV) Collection and assembly of data: None; (V) Data analysis and interpretation: None; (VI) Manuscript writing: All authors; (VII) Final approval of manuscript: All authors.

Correspondence to: William W. Tseng, MD. USC Surgical Oncology, 1510 San Pablo Street, HCC-I, Suite 514, Los Angeles, CA 90033, USA. Email: william.tseng@med.usc.edu.

\begin{abstract}
Retroperitoneal sarcomas (RPS) are rare cancers that often reach massive size before detection. The mainstay of treatment for RPS is surgical resection, and complete resection is the only chance for potential cure. The management of RPS can be challenging and in individual cases, radiation and systemic therapy may be beneficial in both primary and recurrent disease. Further research through multi-institutional collaboration, ideally on a global level, is needed to better understand RPS and optimize management of this disease.
\end{abstract}

Keywords: Retroperitoneal; sarcoma; liposarcoma; leiomyosarcoma

Submitted Jul 04, 2018. Accepted for publication Jul 20, 2018.

doi: $10.21037 /$ cco.2018.07.05

View this article at: http://dx.doi.org/10.21037/cco.2018.07.05

\section{Introduction}

Retroperitoneal sarcomas (RPS) are rare cancers representing only $0.2 \%$ of cancers in adults with an average annual incidence of approximately 2.7 cases per million (1). These tumors originate in the retroperitoneum ("back of the abdomen") and can reach massive size before detection. In fact, the median tumor size on presentation is approximately $20-30 \mathrm{~cm}$. Overall, RPS tend to cause local morbidity rather than metastasize (2). Therefore, the mainstay of treatment for RPS is surgery, with the goal of complete resection.

Surgery for RPS can be challenging due to the massive size of the tumors and potential involvement of adjacent organs and critical structures, all within the confined space in the retroperitoneum. Adequate oncologic resection must be balanced with the anticipated morbidity of surgery. It is recommended that resection be performed by a surgical oncologist with experience in RPS and ideally at a sarcoma center with multidisciplinary expertise in this rare disease. In fact, outcomes for patients with RPS are significantly better when they are treated at a sarcoma center versus a non-specialist center (3).

RPS represent approximately $20 \%$ of a larger group of heterogeneous malignancies known as soft tissue sarcoma. There exist about 50-70 different histologic subtypes of soft tissue sarcoma; however, the most common subtypes in the retroperitoneum are liposarcoma (well differentiated/ dedifferentiated) and leiomyosarcoma. Rarer subtypes including solitary fibrous tumor, undifferentiated pleomorphic sarcoma and malignant peripheral nerve sheath tumor can also be found in the retroperitoneum (4). Although most treatment strategies (e.g., complete resection) can be broadly applied across all histologic subtypes, there is increasing recognition for the importance of subtype-guided management in RPS 


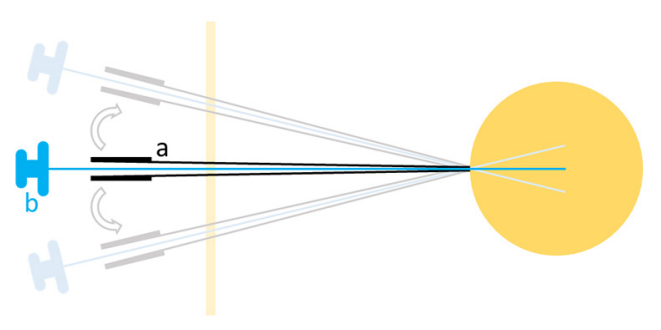

Figure 1 Percutaneous core needle biopsy with coaxial technique performed under CT guidance. With this technique, only one pass of the coaxial sheath (a) to the tumor is performed, followed by multiple passes through the sheath with an automated core biopsy needle (b), which minimizes the risk of seeding. At least 4 cores are taken, changing the angle of the coaxial sheath to sample different areas of the tumor without puncturing the tumor again.

(e.g., systemic therapy in leiomyosarcoma).

\section{Preoperative evaluation}

In general, RPS typically present with nonspecific symptoms such as abdominal discomfort, back pain, and change in bowel or urinary habits. Frequently RPS are found incidentally on routine physical exam or by imaging done for a different reason.

Once detected, if not done already, all RPS should be evaluated with cross-sectional imaging. A good quality, contrast-enhanced computed tomography (CT) scan of the abdomen and pelvis is sufficient in the majority of cases. For tumors with potential spine or nerve root involvement or those that are primarily in the pelvis, a magnetic resonance imaging (MRI) scan may provide additional information. If there is concern or risk for distant metastasis, further staging with a contrast-enhanced CT scan of the chest should also be performed. There is increasing research interest in better defining the role of positron emission tomography (PET) in RPS including its utility in determining tumor grade and potential risk for distant metastasis.

Imaging may provide clues to the histologic subtype (e.g., based on fat content, organ of origin), however ultimately, the definitive diagnosis requires tissue obtained by biopsy. Using an image-guided coaxial technique, percutaneous core needle biopsy is associated with minimal risk of tumor seeding (5) (Figure 1). In a recent study of 255 RPS patients who underwent needle biopsy, only 5 ( $<2 \%)$ developed biopsy site seeding; all of these patients had transabdominal biopsy done without coaxial technique.
Tissue diagnosis by biopsy is important as it rules out other benign and malignant conditions in the retroperitoneum and is mandatory in cases in which neoadjuvant therapy will be given.

Once surgery is being considered, thorough review of the cross-sectional imaging is critical for planning purposes (Figure 2). The surgeon should study the imaging to anticipate the extent of tumor involvement with adjacent organs and structures; however ultimately, this is only definitively determined at the time of surgery. The organs most commonly resected in RPS surgery are kidney and colon, which are removed in approximately half of all RPS patients. In fact, data from recent large series of RPS resections demonstrate that the incidence of nephrectomy and colectomy are $28-55 \%$ and $58 \%$ respectively $(2,6)$.

Preoperative imaging review should also rule out findings that would make a patient unresectable. These findings include multiple peritoneal implants (e.g., sarcomatosis), bilateral renal involvement, extensive spine involvement, extensive mesenteric root involvement, and extensive liver hilar involvement. In such cases, the morbidity and mortality from surgery would outweigh the benefits. For example, resection of the mesenteric root would cause short gut syndrome and require chronic total parenteral nutrition. Patient age and comorbidities should also be taken into account when determining whether it is safe to proceed with surgery (Figure 2).

Surgery for RPS can be challenging and these operations may involve multiple teams, although this practice varies by institution. If needed, preoperative discussion or formal consultation with other surgical teams (e.g., urology for ureteral stent placement to assist with intraoperative identification or vascular surgery for major vessel resection and reconstruction) should be done in advance (7).

\section{Resection of primary disease}

Surgery is the mainstay of treatment in primary RPS for all subtypes. The goal of RPS surgery is complete resection as this is the only chance for cure. Ideally, negative microscopic margins (R0) should be obtained. However, given the massive size of RPS, accurate pathologic assessment of all margins on the resected tumor specimen is difficult to achieve logistically. Therefore, complete resection is often defined as macroscopically negative margins with either negative (R0) or positive (R1) microscopic margins.

Whenever possible, gross tumor should not be left behind (R2). The risk of local recurrence is significantly higher with 

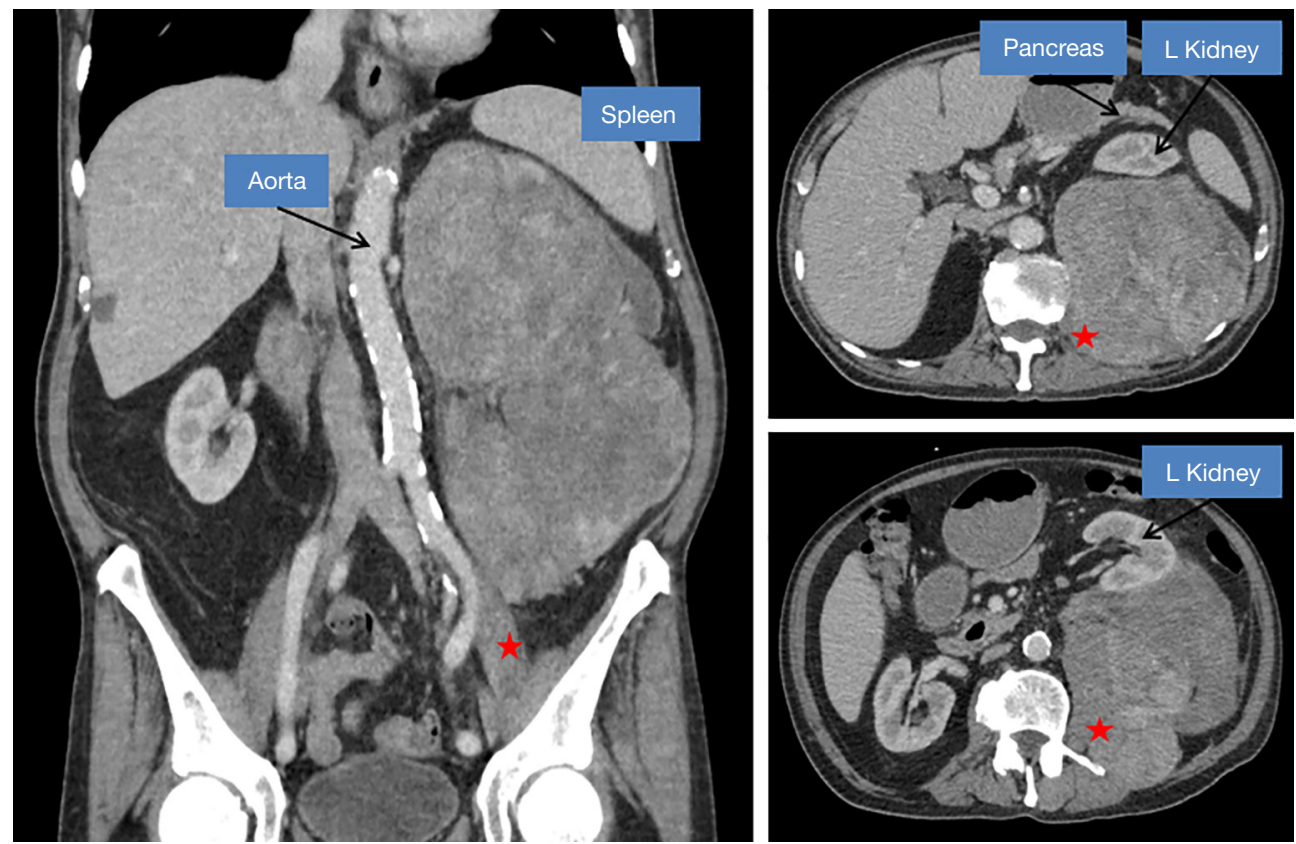

Figure 2 Case example of a patient with a retroperitoneal sarcoma. A frail 81-year-old Asian male with co-morbidities presented with abdominal pain. Imaging demonstrated a large left retroperitoneal soft tissue mass that involves most of the psoas muscle (red star), is adjacent to the spine and lower pole of the left kidney, and is close to the spleen, aorta and left common iliac artery. Biopsy demonstrated high grade, dedifferentiated liposarcoma. At the time of surgery, the well differentiated portion of the tumor was readily apparent, encased the entire left kidney and was adjacent to the left colon mesentery. What is the appropriate extent of resection in this case?

$\mathrm{R} 2$ resection than $\mathrm{R} 0 / \mathrm{R} 1$ resection, with a hazard ratio of 2.81 in a multivariate analysis of a large cohort of RPS patients (2). In addition, intraoperative rupture and piecemeal removal of RPS with violation of the tumor integrity should be avoided as this increases the risk 1.67-fold for local recurrence (2). Fortunately, the technical aspects of surgery for RPS have evolved and in the last two decades, complete resection (R0/ R1) has become feasible and safe in the majority of RPS patients when treated at a sarcoma center (4).

In primary disease, beyond complete resection, there has been active discussion over the optimal extent of resection in regard to organs and structures that are immediately adjacent to the tumor. All sarcoma surgeons agree that at the time of surgery, any adjacent organs and structures with clear tumor involvement should be removed en bloc with the tumor. However, in 2009 the concept of extended or compartmental resection was introduced with two studies from the sarcoma centers in Milan and France $(8,9)$. The authors advocated that even without obvious tumor involvement, whenever feasible, adjacent organs/structures should be systematically resected. This concept is analogous to what is done for extremity soft tissue sarcoma, in which a "margin" of soft tissue is removed en bloc surrounding the tumor. In these two studies, extended resection was associated with improved local control. In one of these studies, Gronchi et al. reported that extended resection performed in 152 pts resulted in a 5-year local recurrence rate of $29 \%$ compared to $48 \%$ in 136 pts who underwent standard resection, in which adjacent organs/structures were removed only if clearly involved (8). Bonvalot et al. reported that extended resection done in 120 RPS patients resulted in a 3.3-fold rate of lower local recurrence compared to 65 patients who had simple complete resection. Three year local recurrence rate was $10 \%$ for extended resection versus $47 \%$ for simple complete resection (9).

The concept of extended resection in primary RPS initially generated controversy, particularly with concerns raised by sarcoma centers in the United States (US). These concerns included the retrospective design of these studies, patient selection bias, intraoperative selection bias, and potentially higher complication rates compared to standard resection (10-12). Intraoperative bias refers to selective resection of organs and the decision to do this based on feasibility and potential morbidity (e.g., psoas 
muscle but not aorta). In addition, US critics pointed out that for both studies, overall survival was equivalent in patients that underwent extended versus standard resection. Longer follow-up data by Gronchi et al. did in fact show improvement in survival for low and intermediate but not high grade RPS (13). These findings along with the identification of microscopic organ invasion in the absence of a clear macroscopic involvement $(14,15)$ have led more recently to an agreed definition of the optimal extent of surgical resection in primary RPS: surgery should be aimed at achieving macroscopically complete resection with a single specimen encompassing the tumor and involved contiguous organs while attempting to minimize microscopically positive margins. Resection of the tumor en bloc with adherent organs even if not overtly involved may be warranted, however preservation of specific organs should be considered on an individual basis. This is often a complex decision and mandates specific expertise in the disease balancing the tumor extent and expected biology with an individual patient's characteristics. Judgement must be used in deciding to resect for example major vessels, liver or pancreas, weighing the potential for local control against the potential for morbidity and dysfunction.

Recently, there is further recognition that specific factors such as histologic subtype significantly affect oncologic outcomes and may be helpful to guide the appropriate extent of resection in RPS. In the retroperitoneum, liposarcoma has a high rate of local recurrence whereas leiomyosarcoma has a high rate of distant metastasis. To highlight this, in the largest multi-institutional study to date, with 1,007 RPS patients the rates of local recurrence after complete resection were $18 \%$ for well differentiated liposarcoma versus $5 \%$ for leiomyosarcoma, whereas the rates for distant metastasis were $0 \%$ versus $55 \%$ (16). This pattern of recurrence based on histologic subtype was also observed in a recent single institution RPS series of 675 patients (6). Therefore, aggressive (extended) resection may be more appropriate in liposarcoma than leiomyosarcoma. However, another study has also suggested that some patients with liposarcoma may have a field defect leading to multifocal locoregional disease regardless of the extent of surgery, suggesting that in these patients, aggressive resection may be of limited benefit (17).

\section{The role of radiation and systemic therapy for RPS}

In some RPS patients, in addition to surgery, there may be an additional role for nonsurgical therapies, including radiation or systemic therapy; however, these are not standardized at this point. The decision for radiation or systemic therapy in RPS remains individualized and should be made in the setting of multidisciplinary team discussion.

A recent analysis of the NCDB did show a benefit with the addition of radiotherapy to surgery in RPS (18). In this study a case-control propensity score match was performed and patients receiving radiation pre- and post-operatively had improved overall survival compared to surgery alone (62\% vs. $54 \% 5$-year survival for pre-op vs. surgery and $60 \%$ vs. $52 \%$ for the post-op vs. surgery). This is in contrast however, to other retrospective studies and non-randomized trials that have not established improved survival and in fact, had mixed results for local recurrence (19-23). When radiation is considered for RPS, preoperative delivery is preferred as this limits damage to uninvolved organs (e.g., small bowel). To date, two randomized clinical trials, ACSOG Z9031 and the subsequent EORTC 62092-22092 (STRASS) have attempted to evaluate the benefit of neoadjuvant radiotherapy over surgery alone. The ACOSOG trial closed early due to poor accrual and the preliminary result of the STRASS study did not demonstrate an improvement in survival parameters at the interim analysis (unpublished data). Subanalysis by histologic subtype for the STRASS trial is pending.

The use of systemic therapy including chemotherapy for RPS also remains controversial. Studies evaluating the role of conventional chemotherapy in soft tissue sarcomas have included low numbers of retroperitoneal tumors or they have been excluded all together $(24,25)$. Therefore, extrapolating the findings to RPS is difficult. In the adjuvant setting, tumor subtypes that have suggested benefit are those that are high grade (e.g., dedifferentiated but not well differentiated liposarcoma) or those with high metastatic potential (e.g., leiomyosarcoma). The use of chemotherapy in the neoadjuvant setting has been utilized to evaluate response to therapy and when a decrease in tumor size would provide a surgical advantage. Neoadjuvant chemotherapy has been shown to be safe, but improvement in local control or survival versus surgery alone have not yet been specifically studied $(26,27)$.

The standard conventional first-line chemotherapeutic regimen for soft tissue sarcoma is doxorubicin or doxorubicin/ifosfamide. In leiomyosarcoma specifically, many centers use the combination of doxorubicin with dacarbazine, given the higher response rate and disease control relative to doxorubicin alone or in combination with 
ifosfamide. Recently there have been several new systemic therapies that have shown efficacy in specific histologic subtypes. These include: pazopanib for non-adipocytic tumors, trabectedin for liposarcoma and leiomyosarcoma, eribulin for liposarcoma, and palbociclib for liposarcoma (28-32). While none have shown significant improvement over standard regimens they have similar outcomes with fewer toxicities. The benefit of these new subtype-specific systemic therapies in RPS in either the neoadjuvant or adjuvant setting remains to be evaluated.

Immunotherapy, in particular immune checkpoint blockade, is being explored in soft tissue sarcoma and tumor responses have been reported to be subtype specific (33). Tseng et al. reported translational data to support feasibility of immunotherapy in retroperitoneal liposarcoma; however to our knowledge, the therapeutic efficacy has not yet been formally evaluated in RPS patients $(34,35)$.

\section{Resection of recurrent disease}

For patients with locally recurrent RPS, surgery is still considered the mainstay of treatment, when feasible. There is data to support that complete resection in patients with recurrent RPS improves survival. As an example, in a study of 219 patients with locally recurrent RPS, the twoand five-year survival rates with resection were $73 \%$ and $43 \%$, vs. $43 \%$ and $11 \%$ without resection (36). However, the decision-making process to resect and the timing of resection with potential integration of nonsurgical therapies (e.g., radiation and systemic therapy) can be challenging. In recurrent disease, even more so than in primary disease, case discussion in a multidisciplinary team setting is critical.

Operating on a patient with recurrent RPS can also be more technically challenging than operating on a patient with primary disease. The anatomy and natural tissue planes are distorted and frequently lysis of bowel adhesions is needed, with the higher risk of complications such as enterotomy and postoperative ileus. In general, each subsequent recurrence of RPS is associated with lower rates of complete resection and worse clinical outcome (37).

Appropriate selection of patients with recurrent RPS who are most likely to benefit from re-resection is therefore key. Important considerations which may favor re-resection include prior complete resection (no gross residual disease), no intraoperative tumor rupture or prior piecemeal resection, low-grade tumors (e.g., well differentiated liposarcoma), longer disease-free interval, and unifocal rather than multifocal locoregional disease $(36,38,39)$. For retroperitoneal liposarcoma specifically, an initial period of observation before deciding to resect may be worthwhile. One study by Park et al. found that the growth rate of recurrent disease was strongly associated with outcomes after resection. Patients with tumors that grew faster than $0.9 \mathrm{~cm}$ per month did not benefit from re-resection, with poor disease-specific survival on par with patients who were not resected (40).

In patients who are not candidates for resection, percutaneous techniques such as radiofrequency ablation can also be considered. This has been reported in several cases of RPS but to our knowledge has not been systematically studied (41-43).

\section{Palliative resection}

In very selective situations, palliative resection of RPS may be considered to relieve severe symptoms. Surgery in these cases may encompass debulking (R2) or piecemeal resection depending on the individual patient. Palliative RPS resection may also be indicated in some patients with known metastatic disease. In these patients, one important consideration is the interruption of systemic therapy before and after surgery that could potentially result in disease progression at metastatic sites.

The decision-making process for palliative RPS resection is complex and cases should certainly be discussed among multidisciplinary team members. One specific challenge in selecting appropriate RPS patients for palliative resection is determining whether surgery will truly offer significant and durable symptom relief. For example, in one study, although $54 \%$ of gastrointestinal obstructive symptoms were relieved at 30 days by palliative surgery for sarcoma, only $23 \%$ of patients remained symptom free at 100 days (44).

\section{Prognosis and surveillance}

Following resection of RPS for curative intent, the most important prognostic factor is the completeness of resection. Other factors including age, tumor size, histologic subtype, grade and multifocality are also important. In fact, nomograms have been developed specifically for RPS which incorporate these and other factors to predict outcome with respect to recurrence and survival. Gronchi et al. incorporated data from 523 RPS patients who underwent resection at 3 sarcoma centers in the US and Europe to create such a nomogram which was then validated in the same study at a $4^{\text {th }}$ sarcoma center (45). This prognostic RPS nomogram is now free and available online and 
through the smartphone application known as "Sarculator": https://www.sarculator.com/.

In all patients with RPS resection, close surveillance is very important. Recommendations are available from consensus groups (e.g., NCCN, ESMO), although these guidelines are not universally accepted (46). In general, after surgery, a contrast-enhanced CT scan is recommended to confirm absence of residual disease and to serve as a new baseline. Subsequently, along with history and physical, CT scans should be performed at regular intervals every 3-6 months for 2-3 years, then every 6 months until the 5 -year point, and then annually. RPS does have the potential for late recurrence. In fact, local recurrences at 10 years and beyond after surgery have been reported (2).

Postoperative surveillance should also be tailored according to the patient's histologic subtype and anticipated pattern of recurrence. As discussed previously, patients with well differentiated liposarcoma are at risk for local recurrence but these tumors do not metastasize (CT chest may not be necessary if the diagnosis is confirmed). In contrast, patients with leiomyosarcoma are at high risk for distant metastasis but relatively low risk for local recurrence, while patients with dedifferentiated liposarcoma are at risk for both local and distant recurrence. Patients with multifocal disease (more than one tumor in the retroperitoneum) which is more commonly seen in liposarcoma, are at especially higher risk of local recurrence after resection $(17,47)$. In these patients, initial shorter time intervals between scans may be warranted, however this has never been formally studied. Currently, there are no laboratory tests to detect recurrence prior to development of visible disease seen by cross sectional imaging; however, there is active investigation into this (e.g., serum biomarkers) for RPS including liposarcoma (48).

\section{Opportunities for collaboration and conclusions}

Further research is necessary in RPS and given the rarity of the disease, multi-institutional collaboration is essential. An example of such a recent collaborative effort is the Trans-Atlantic Retroperitoneal Sarcoma Working Group (TARPSWG), formed in 2013 between US and European sarcoma centers. Through the efforts of TARPSWG, surgical technique papers and consensus guidelines for primary, recurrent and metastatic disease have been published (49-52). In addition, original research has been made possible for broadly relevant issues (e.g., morbidity of RPS resection) (36) and rare situations in this rare disease (e.g., Whipple procedure in RPS) (53). Current, ongoing projects within TARPSWG include a prospective clinical database and shared tissue bank for RPS.

Continued organized collaboration among other sarcoma centers around the world beyond "Trans-Atlantic" is critical to move forward in our understanding and management of RPS. Specifically, incorporating sarcoma centers in other countries (e.g., Asia) offers several distinct advantages:

First, collaboration would allow global validation of clinical practice strategies across different patient populations for the same disease. Multiple studies have looked at prognostic factors in Chinese RPS patients and have confirmed the importance of completeness of resection, tumor grade, and histologic subtype, similar to findings already reported with US and European RPS patients (54-56). Chou et al. specifically performed validation of Gronchi's prognostic nomogram for RPS in an Eastern (Taiwanese) cohort of patients and found an excellent concordance score (0.72), suggesting broad applicability of this clinical tool (57). Sato et al. reported their case series of Japanese retroperitoneal liposarcoma patients that benefited from repeated resections (58), a practice also done among US and European patients that deserves further formal validation (59).

Second, through collaboration, unique observations or novel treatment methods can be studied in a combined fashion with the goal of improving outcomes for all RPS patients globally. Setsu et al. reported a case series of five Japanese patients with genetically confirmed (DDIT3 translocation-positive), primary myxoid liposarcoma arising in the retroperitoneum: a rare finding (60). Yu et al. have reported accumulating experience with adjuvant radiation (tomotherapy) using tissue expanders in Korean patients with resected RPS $(61,62)$. Both of these are unique situations that should be further studied in Western RPS patients as well.

Third, global collaboration in RPS can also potentially detect interesting variations in disease biology. For example, Miao et al. identified a single-nucleotide polymorphism in folylpolyglutamate synthase that was strongly associated with increased risk for retroperitoneal liposarcoma in a cohort of Chinese patients (63). She et al. found that expression of CREPT (cell cycle-related and expressionrelated protein in tumor) was correlated with earlier recurrence and worse survival in Chinese patients with retroperitoneal leiomyosarcoma (64). The central question is whether these clinical biomarkers and others are also seen in non-Chinese RPS and if not, the implications for disease biology would need further investigation. 
Overall, given the rarity of RPS, through global multiinstitutional collaboration, research can be expedited compared to research done at a single institution alone. Logistically, global collaboration would require complex coordination and would in itself, present unique challenges. For example, standardization of fundamental clinical practice strategies (e.g., extent of surgery or systemic therapy for a given histologic subtype) would have to be discussed and mutually agreed upon, ideally based on available data. This would actually be a remarkable opportunity to promote discussion among sarcoma centers in different countries. Once a research study is initiated, sharing data and especially tissue for research across sarcoma centers may also be logistically challenging but not impossible.

In conclusion, surgery with complete resection is the mainstay of treatment in RPS and the only potential chance for cure. As this review highlights, the management of RPS can be challenging and integration of nonsurgical therapies (e.g., radiation and systemic therapy) may be beneficial in individual cases in both primary and recurrent disease. With further research through multi-institutional collaboration, ideally on a global level, we can better understand RPS and optimize management for this rare and challenging disease.

\section{Acknowledgements}

None.

\section{Footnote}

Conflicts of Interest: The authors have no conflicts of interest to declare.

\section{References}

1. Porter GA, Baxter NN, Pisters P. Retroperitoneal sarcoma: A population-based analysis of epidemiology, surgery, and radiotherapy. Cancer 2006;106:1610-6.

2. Gronchi A, Strauss DC, Miceli R, et al. Variability in patterns of recurrence after resection of primary retroperitoneal sarcoma (RPS): A report on 1007 patients from the Multi-institutional Collaborative RPS Working Group. Ann Surg 2016;263:1002-9.

3. Toulmonde M, Bonvalot S, Méeus P, et al. Retroperitoneal sarcomas: Patterns of care at diagnosis, prognostic factors and focus on main histological subtypes: A multicenter analysis of the French Sarcoma Group. Ann Oncol 2014;25:735-42.
4. Tseng WW, Seo HJ, Pollock RE, et al. Historical perspectives and future directions in the surgical management of retroperitoneal sarcoma. J Surg Oncol 2018;117:7-11.

5. Van Houdt WJ, Schrijver AM, Cohen-Hallaleh RB, et al. Needle tract seeding following core biopsies in retroperitoneal sarcoma. Eur J Surg Oncol 2017;43:1740-5.

6. Tan MC, Brennan MF, Kuk D, et al. Histology-based classification predicts pattern of recurrence and improves risk stratification in primary retroperitoneal sarcoma. Ann Surg 2016;263:593-600.

7. Tseng WW, Wang SC, Eichler CM, et al. Complete and safe resection of challenging retroperitoneal tumors: Anticipation of multi-organ and major vascular resection and use of adjunct procedures. World J Surg Oncol 2011;9:143.

8. Gronchi A, Lo Vullo S, Fiore M, et al. Aggressive surgical policies in a retrospectively reviewed single-institution case series of retroperitoneal soft tissue sarcoma patients. J Clin Oncol 2009;27:24-30.

9. Bonvalot S, Rivoire M, Castaing M, et al. Primary retroperitoneal sarcomas: A multivariate analysis of surgical factors associated with local control. J Clin Oncol 2009;27:31-7.

10. Pisters PW. Resection of some but not all clinically uninvolved adjacent viscera as part of surgery for retroperitoneal soft tissue sarcomas. J Clin Oncol 2009;27:6-8.

11. Gronchi A, Pollock R. Surgery in retroperitoneal soft tissue sarcoma: A call for a consensus between Europe and North America. Ann Surg Oncol 2011;18:2107-10.

12. Crago AM. Extended surgical resection and histology in retroperitoneal sarcoma. Ann Surg Oncol 2015;22:1401-3.

13. Gronchi A, Miceli R, Colombo C, et al. Frontline extended surgery is associated with improved survival in retroperitoneal low- to intermediate-grade soft tissue sarcomas. Ann Oncol 2012;23:1067-73.

14. Mussi C, Colombo P, Bertuzzi A, et al. Retroperitoneal sarcoma: Is it time to change the surgical policy? Ann Surg Oncol 2011;18:2136-42.

15. Fairweather M, Wang J, Jo VY, et al. Incidence and adverse prognostic implications of histopathologic organ invasion in primary retroperitoneal sarcoma. J Am Coll Surg 2017;224:876-83.

16. Gronchi A, Miceli R, Allard MA, et al. Personalizing the approach to retroperitoneal soft tissue sarcoma: Histologyspecific patterns of failure and postrelapse outcome 
after primary extended resection. Ann Surg Oncol 2015;22:1447-54.

17. Tseng WW, Madewell JE, Wei W, et al. Locoregional disease patterns in well-differentiated and dedifferentiated retroperitoneal liposarcoma: Implications for the extent of resection? Ann Surg Oncol 2014;21:2136-43.

18. Nussbaum DP, Rushing CN, Lane WO, et al. Preoperative or postoperative radiotherapy versus surgery alone for retroperitoneal sarcoma: A case-control, propensity score-matched analysis of a nationwide clinical oncology database. Lancet Oncol 2016;17:966-75.

19. Catton CN, O'Sullivan B, Kotwall C, et al. Outcome and prognosis in retroperitoneal soft tissue sarcoma. Int J Radiat Oncol Biol Phys 1994;29:1005-10.

20. Hassan I, Park SZ, Donohue JH, et al. Operative management of primary retroperitoneal sarcomas: a reappraisal of an institutional experience. Ann Surg 2004:239:244-50.

21. Le Péchoux C, Musat E, Baey C, et al. Should adjuvant radiotherapy be administered in addition to frontline aggressive surgery (FAS) in patients with primary retroperitoneal sarcoma? Ann Oncol 2013;24:832-7.

22. Jones JJ, Catton CN, O'Sullivan B, et al. Initial results of a trial of preoperative external-beam radiation therapy and postoperative brachytherapy for retroperitoneal sarcoma. Ann Surg Oncol 2002;9:346-54.

23. Kelly KJ, Yoon SS, Kuk D, et al. Comparison of perioperative radiation therapy and surgery versus surgery alone in 204 patients with primary retroperitoneal sarcoma: A retrospective 2-institution study. Ann Surg 2015;262:156-62.

24. Gortzak E, Azzarelli A, Buesa J, et al. A randomised phase II study on neo-adjuvant chemotherapy for 'high-risk' adult soft-tissue sarcoma. Eur J Cancer 2001;37:1096-103.

25. Woll PJ, Reichardt P, Le Cesne A, et al. Adjuvant chemotherapy with doxorubicin, ifosfamide, and lenograstim for resected soft-tissue sarcoma (EORTC 62931): a multicentre randomised controlled trial. Lancet Oncol 2012;13:1045-54.

26. Meric F, Milas M, Hunt KK, et al. Impact of neoadjuvant chemotherapy on postoperative morbidity in soft tissue sarcomas. J Clin Oncol 2000;18:3378-83.

27. Almond LM, Gronchi A, Strauss D, et al. Neoadjuvant and adjuvant strategies in retroperitoneal sarcoma. Eur J Surg Oncol 2018;44:571-9.

28. van der Graaf WT, Blay JY, Chawla SP, et al. Pazopanib for metastatic soft-tissue sarcoma (PALETTE): A randomised, double-blind, placebo-controlled phase 3 trial. Lancet 2012;379:1879-86.
29. Demetri GD, von Mehren M, Jones RL, et al. Efficacy and safety of trabectedin or dacarbazine for metastatic liposarcoma or leiomyosarcoma after failure of conventional chemotherapy: Results of a phase III randomized multicenter clinical trial. J Clin Oncol 2016;34:786-93.

30. Schöffski P, Chawla S, Maki RG, et al. Eribulin versus dacarbazine in previously treated patients with advanced liposarcoma or leiomyosarcoma: A randomised, openlabel, multicentre, phase 3 trial. Lancet 2016;387:1629-37.

31. Dickson MA, Schwartz GK, Keohan ML, et al. Progression-free survival among patients with welldifferentiated or dedifferentiated liposarcoma treated with CDK4 inhibitor palbociclib: A phase 2 clinical trial. JAMA Oncol 2016;2:937-40.

32. Stoeckle E, Coindre JM, Bonvalot S, et al. Prognostic factors in retroperitoneal sarcoma: A multivariate analysis of a series of 165 patients of the French Cancer Center Federation Sarcoma Group. Cancer 2001;92:359-68.

33. Tawbi HA, Burgess M, Bolejack V, et al. Pembrolizumab in advanced soft-tissue sarcoma and bone sarcoma (SARC028): A multicentre, two-cohort, single-arm, openlabel, phase 2 trial. Lancet Oncol 2017;18:1493-501.

34. Tseng WW, Malu S, Zhang M, et al. Analysis of the intratumoral adaptive immune response in well differentiated and dedifferentiated retroperitoneal liposarcoma. Sarcoma 2015;2015:547460.

35. Tseng WW, Demicco EG, Lazar AJ, et al. Lymphocyte composition and distribution in inflammatory, welldifferentiated retroperitoneal liposarcoma: clues to a potential adaptive immune response and therapeutic implications. Am J Surg Pathol 2012;36:941-4.

36. MacNeill AJ, Miceli R, Strauss DC, et al. Postrelapse outcomes after primary extended resection of retroperitoneal sarcoma: A report from the Trans-Atlantic RPS Working Group. Cancer 2017;123:1971-8.

37. Lewis JJ, Leung D, Woodruff J, et al. Retroperitoneal softtissue sarcoma: Analysis of 500 patients treated and followed at a single institution. Ann Surg 1998;228:355-65.

38. Hamilton TD, Cannell AJ, Kim M, et al. Results of resection for recurrent or residual retroperitoneal sarcoma after failed primary treatment. Ann Surg Oncol 2017;24:211-8.

39. Grobmyer SR, Wilson JP, Apel B, et al. Recurrent retroperitoneal sarcoma: Impact of biology and therapy on outcomes. J Am Coll Surg 2010;210:602-8, 608-10.

40. Park JO, Qin LX, Prete FP, et al. Predicting outcome by growth rate of locally recurrent retroperitoneal liposarcoma: The one centimeter per month rule. Ann 
Surg 2009;250:977-82.

41. Keil S, Bruners P, Brehmer B, et al. Percutaneous radiofrequency ablation for treatment of recurrent retroperitoneal liposarcoma. Cardiovasc Intervent Radiol 2008;31 Suppl 2:S213-6.

42. Pawlik TM, Vauthey JN, Abdalla EK, et al. Results of a single-center experience with resection and ablation for sarcoma metastatic to the liver. Arch Surg 2006;141:537-43.

43. Qin Z, Zeng J, Liu G, et al. Irreversible electroporation ablation of an unresectable fibrous sarcoma with 2 electrodes: A case report. Technol Cancer Res Treat 2017:1533034617711530. [Epub ahead of print].

44. Yeh JJ, Singer S, Brennan MF. Effectiveness of palliative procedures for intra-abdominal sarcomas. Ann Surg Oncol 2005;12:1084-9.

45. Gronchi A, Miceli R, Shurell E, et al. Outcome prediction in primary resected retroperitoneal soft tissue sarcoma: Histology-specific overall survival and disease-free survival nomograms built on major sarcoma center data sets. J Clin Oncol 2013;31:1649-55.

46. Zaidi MY, Canter R, Cardona K. Post-operative surveillance in retroperitoneal soft tissue sarcoma: The importance of tumor histology in guiding strategy. J Surg Oncol 2018;117:99-104.

47. Anaya DA, Lahat G, Liu J, et al. Multifocality in retroperitoneal sarcoma: a prognostic factor critical to surgical decision-making. Ann Surg 2009;249:137-42.

48. Casadei L, Calore F, Creighton CJ, et al. Exosomederived miR-25-3p and miR-92a-3p stimulate liposarcoma progression. Cancer Res 2017;77:3846-56.

49. Trans-Atlantic RPS Working Group. Management of primary retroperitoneal sarcoma (RPS) in the adult: A consensus approach from the Trans-Atlantic RPS Working Group. Ann Surg Oncol 2015;22:256-63.

50. Trans-Atlantic RPS Working Group. Management of Recurrent Retroperitoneal Sarcoma (RPS) in the Adult: A Consensus Approach from the Trans-Atlantic RPS Working Group. Ann Surg Oncol 2016;23:3531-40.

51. Trans-Atlantic RPS Working Group. Management of metastatic retroperitoneal sarcoma: a consensus approach from the Trans-Atlantic Retroperitoneal Sarcoma Working Group (TARPSWG). Ann Oncol 2018;29:857-71.

52. Bonvalot S, Raut CP, Pollock RE, et al. Technical considerations in surgery for retroperitoneal sarcomas: Position paper from E-Surge, a master class in sarcoma surgery, and EORTC-STBSG. Ann Surg Oncol 2012;19:2981-91.

53. Tseng WW, Tsao-Wei DD, Callegaro D, et al.
Pancreaticoduodenectomy in the surgical management of primary retroperitoneal sarcoma. Eur J Surg Oncol 2018;44:810-5.

54. Chen CQ, Yin L, Peng CH, et al. Prognostic factors of retroperitoneal soft tissue sarcomas: analysis of 132 cases. Chin Med J (Engl) 2007;120:1047-50.

55. Li Q, Zhuang R, Zhu J, et al. Prognostic factors in patients with recurrent or metastatic retroperitoneal leiomyosarcoma. Future Oncol 2015;11:1759-66.

56. Zhao X, Li P, Huang X, Chen L, et al. Prognostic factors predicting the postoperative survival period following treatment for primary retroperitoneal liposarcoma. Chin Med J (Engl) 2015;128:85-90.

57. Chou YS, Liu CY, Chang YH, et al. Prognostic factors of primary resected retroperitoneal soft tissue sarcoma: Analysis from a single asian tertiary center and external validation of Gronchi's nomogram. J Surg Oncol 2016;113:355-60.

58. Sato T, Yamaguchi T, Azekura K, et al. Repeated resection for intra-abdominal and retroperitoneal liposarcomas: Long-term experience in a single cancer center in Japan. Int Surg 2006;91:267-71.

59. Bagaria SP, Gabriel E, Mann GN. Multiply recurrent retroperitoneal liposarcoma. J Surg Oncol 2018;117:62-8.

60. Setsu N, Miyake M, Wakai S, et al. Primary retroperitoneal myxoid liposarcomas. Am J Surg Pathol 2016;40:1286-90.

61. Yu JI, Lim DH, Park HC, et al. Effectiveness and safety of tissue expander for adjuvant helical tomotherapy in curatively resected retroperitoneal sarcoma. Anticancer Res 2018;38:2447-53.

62. Yu JI, Lim DH, Park HC, et al. Clinical outcomes of tissue expanders on adjuvant radiotherapy of resected retroperitoneal sarcoma. Medicine (Baltimore) 2016;95:e4123.

63. Miao C, Liu D, Zhang F, et al. Association of FPGS genetic polymorphisms with primary retroperitoneal liposarcoma. Sci Rep 2015;5:9079.

64. She Y, Liang J, Chen L, et al. CREPT expression correlates with poor prognosis in patients with retroperitoneal leiomyosarcoma. Int J Clin Exp Pathol 2014;7:6596-605.

Cite this article as: Wang J, Grignol VP, Gronchi A, Luo CH, Pollock RE, Tseng WW. Surgical management of retroperitoneal sarcoma and opportunities for global collaboration. Chin Clin Oncol 2018;7(4):39. doi: 10.21037/ cco.2018.07.05 